\title{
Semantic Knowledge Based Approach for Product Maintenance Support
}

\author{
I.Sanya ${ }^{1}$, E.Shehab ${ }^{1}$, R.Roy ${ }^{1}$, O.Houseman ${ }^{2}$, M.Jonik $^{2}$ \\ ${ }^{1}$ Manufacturing Department, Cranfield University, Cranfield, Bedford, MK43 0AL, UK \\ ${ }^{2}$ TechModal, Bristol, UK \\ \{i.o.sanya; e.shehab; r.roy\}@cranfield.ac.uk
}

\begin{abstract}
The purpose of this paper is to use semantic technology to represent the knowledge for product maintenance. Companies are begining to understand the importance of utilising an effective approach to manage existing knowledge in order to increase their intellectual capital. However, one of the main constraints that have hindered the solution in resolving technical problems has been the efficient access to knowledge and expertise. Therefore, there is need for enhancing the management and maintenance of knowledge through a semantic based approach. This research project adopts a qualitative research approach and a five-phase research programme methodology. This research project has highlighted that semantic technology enhances the reusability, flexibility and maintainability of knowledge and its management.
\end{abstract}

\section{Keywords:}

Semantics; Ontologies; Concepts; Rules; Knowledge Representation;

\section{INTRODUCTION}

The management of knowledge is now quickly becoming an important business activity for many companies as they realise that in order to remain competitive, there is need for effectively managing intellectual resources. However, there is lack of 'linguistics' within the way conventional systems communicate and companies are beginning to realise the importance of 'semantics' within IT systems.

For this reason, the theme of this research project will focus on identifying and developing a prototype application that adopts the use of semantic technology for knowledge management purposes. The semantic based prototype involves the transformation of an existing validated manual for bicycle diagnosis repair. The outcome of this research project is the representation of this manual embedded within a semantic based application for product maintenance support.

The capture of complex knowledge and maintenance diagnosis has always been an essential factor that has contributed to the success of companies as they realise that their most valuable resource is the knowledge of its people. However, one of the main constraints that have hindered the solution in resolving technical problems has been the efficient access to knowledge and expertise due to experts that are bounded by their geographical location or the sudden departure of subject matter experts. As a result of this, current engineers end up spending large amount of time to find a solution to previously resolved problems.

This leads to increased time-to-fix, downtime, and substantial unnecessary cost increase. A potential solution to this problem is the use of a knowledge based system which will reproduce knowledge from human experts utilising latest technologies such as semantics, declarative rules and ontologies.

A prototype demonstrator is then needed that will quantify the use of semantic technology within this field. A common solution has been the use of knowledge based systems which allows for knowledge reproduction. However, current knowledge based systems lacks meaning in communication which means a more efficient and effective way is needed for managing and maintaining knowledge in order to correct defects and improve system performance.

Thus, the main aim of this research project is to develop a prototype tool that will utilise semantic technology for the management and maintenance of knowledge in order to demonstrate and quantify the capability of this technology within the defence equipment support market.

\section{RELATED LITERATURE}

In today's world, any discussion of knowledge leads to understanding how knowledge is defined. In practice, knowledge is defined as starting at data, encompassing information and ending at knowledge. According to Grover and Davenport [1], knowledge is known to have the highest value in human contribution. It is the greatest relevance to making decisions and actions and it is also the most difficult content type to manage because it exists in people's mind tacitly.

Thus, there is need for effective knowledge capture. The National Health Service [2] suggests that the main purpose of knowledge capture is to acquire an expert's decision making process in great detail so that someone else could repeat the same set of process and establish the same results/conclusions.

The National Health Service [2] furthermore suggests that one of the best ways to capture tacit knowledge is using one-to-one, face-to-face interviews with experts. However, Milton [3] suggests other effective techniques such as protocol generation technique which is used to establish a record of behaviour, protocol analysis technique which is used for the identification of knowledge objects within a protocol and hierarchical techniques which is used to create, review and modify hierarchical knowledge in form of ladders.

O'Hara and Shadbolt [4] stated that the purpose of having so many knowledge elicitation techniques remains in the fact that experts possess various forms of tacit knowledge. It is essential to utilise the appropriate 
technique in order to access and capture the various types of knowledge. This process is referred to as the "differential access hypothesis" and has been implemented in real world settings with supporting evidence [3, 4].

Knowledge Based Systems are part of Al applications seeing as they emulate the "thinking processes" taken by humans in order to solve broad classes of problems [5]. Most research states that the main difference in which knowledge based systems differs from conventional systems is the approach taken to solve a problem. Conventional systems solve problems via algorithms, whereas KBS uses heuristics which is intended to increase the probability of solving a problem $[6,7]$. The benefits of KBS has agreed by most authors includes time saving $[7,8,9]$, quality improvement $[7,8,9,10]$, applicability of practical knowledge $[7,8,11]$ and consistency $[7,10,11]$.

Furthermore, there has been vast amount of research within the area of computer based knowledge representation. It has been established that computers are a great way of storing and retrieving information. However, many have wondered about the current advancements with the science behind computer information [12].

Barski [12] argues that the scientist has not contributed enough in the world of knowledge representation and software and stated that the third revolution of computer's will be the era of the scientist which will allow computers to represent knowledge using integral calculus, theorem proving, linguistics (semantics), and other form of scientific distinct feature.

Furthermore, in today's world, the meaning of web content is not machine-accessible which means there is lack of semantics [13]. Semantics is defined as the study of meaning in communication. There is need for knowledge representation in semantics. Knowledge representation in semantics allows for the description of the problem domain, with concepts and the relations between those concepts. It allows for a shared agreement of meanings. All semantic based knowledge representation techniques must utilise "subject based classification". This is any form of content classification that groups objects by their subjects.

Semantic technology relies on the ontology model. This is a model that represents a set of concepts and the relationships between these concepts. This extends the subject based classification approaches due to its open vocabularies and open relationship types. The use of ontologies to represent complex knowledge has become common in government bodies such as NASA and UN [14].

More current advancements on semantic technology have occurred within the enterprise space. Semantic technology is used in order to integrate information and improve efficiency. Davies et al [15] stated that BT's own fusion of billing and sales in one department has allowed for £2million annual savings and ongoing semantic innovation is being considered within this sector.

Davies et al [15] suggested that companies such as Hakia, Siderean and Ontotext are carrying out underlying research within the area of semantic search. Also, within the area of information and process integration, there are companies such as Metatomix, Ontoprise and Radar Networks who just recently announced its "Twine semantic social networks offering"

Google also announced that it was changing the way in which search engines interpret words entered by users which highlights the importance of semantics [16].

Research has proved recently that semantic technology has been used within medical centres [17] and also within the financial Industry [18]. This type of technology is highly required within the defence equipment support market for the capture \& maintenance of complex knowledge; however, semantic technology is not being utilised. This is where the weak links exists and the outcome of this research project will evaluate \& validate the use of this technology within the defence equipment support market by developing an equipment diagnosis prototype as a demonstrator to quantify the use of semantic technology.

Many Researchers [19] argue that today's equipments incur high cost for maintenance due to its complexity. Therefore, it is necessary to enhance modern maintenance management systems. The conventional condition-based maintenance (CBM) is usually used to reduce maintenance activities and operates according to the indication of an equipment condition. Yam et al, [19] illustrates that a recent study of maintenance management practices demonstrates that the three major problems facing modern engineering plants are the reduction of high inventory cost for spare parts, preplanning maintenance work for complex equipment under a complex environment and avoiding the risk of major failure and eliminating catastrophic circumstances that could occur to an equipment or system.

Fuchs et al [20] supports the view of most researchers about the complexity of industrial systems and the need for highly capable maintenance systems for monitoring data. The key issue highlighted by this paper suggests that there is lack of linguistics in representing and querying data from multiple sources.

The literature review has highlighted that there is lack of research projects focused on the application of semantic technology within maintenance support. Therefore, this research project has attempted to fill in this research gap by developing a semantic based prototype for this purpose.

\section{RESEARCH METHODOLOGY}

\subsection{The Case Study Company}

TechModal was founded in 2005 by $\mathrm{PhD}$ students researching semantic technology and cost engineering techniques within the aerospace sector. The company was formed to utilise the latest web technologies for practical solutions within decision support. Having researched the field of semantic technology at $\mathrm{PhD}$ level and implemented semantic technology applications in industry, TechModal have selected a world leading commercial semantic platform provider company known as Ontoprise as the most efficient framework on which to build semantic applications. Techmodal costing activities within the defence sector includes the Cost Capability Trade-Off, Value for Money Comparison (VFMC), Cost Benefit Analysis and Cost Effectiveness Analysis [21].

\subsection{The Research Methodology}

Figure 1 illustrates the adopted research methodology. The research project commenced with a familiarisation stage which clearly defined the project aim and objectives. An extensive literature review was used in order to identify research gaps and understand the review of literature cases on use of semantics and maintenance support. The output of this phase clearly identified the current use of semantics to manage and maintain knowledge. The second phase was for the researcher to undergo adequate training on the semantic platform proposed by the sponsoring company (Techmodal). Understanding how to utilise this semantic 
based application for the purpose of declaring concepts, rules and ontologies for a problem domain was essential in achieving the aim and objectives of the project. The output from this phase enhanced the researcher's familiarisation with the basics of developing a semantic based application for a specific problem domain. This stage was an ongoing activity seeing as new knowledge was discovered as the researcher continued to utilise the semantic based platform.

The platform known as SemanticGuide was developed by Ontoprise [22] and this platform has been utilised in this project. This platform is an ontology based intelligent advisory system which allows for knowledge automation. SemanticGuide [22] uses the idea of concepts which is referred to as the domain model ontology, rules (operational model ontology) and then an export mechanism to publish the application onto the web. This approach is used to model a problem domain.

The third phase was to capture and represent knowledge effectively using knowledge representation techniques such as UML (Unified Modelling Language) use case diagram and ontological knowledge representation techniques (Taxonomy). This phase also involved collecting and understanding the validated manual repair for a bicycle in order to transform this knowledge model into a semantic based platform.

The fourth phase was to develop the semantic based prototype. The development of this prototype established the key aspects and benefits of semantic technology Furthermore, the contribution to knowledge and application of results were identified.

Lastly, phase five validates the developed prototype and ensures the appropriate dissemination of the project results. The qualitative phenomenological study is an interesting study for this research project due to the fact that ontologies are used to model the semantic based prototype for the domain area and an ontology is described as the study of being and existence.

The quantitative approach was not adopted because the knowledge required has already been captured and validated within the Barnett's bicycle repair manual handbook. The qualitative approach enhanced the richness of what was being achieved and the researcher became subjectively immersed in the subject matter.

\section{THE PROTOTYPE DEVELOPMENT}

After capturing and representing knowledge from the validated bicycle diagnosis repair, the semantic based prototype was developed using the semantic platform proposed by the sponsoring company. Semantic Guide uses the idea of rules, concepts and ontologies to model the problem domain.

\subsection{Module 1: Maintenance Support}

The development of the semantic based prototype for maintenance support commenced using SemanticGuide. Figure 2 illustrates the system architecture for SemanticGuide. This demonstrates that SemanticGuide has two types of system engineers. These are the knowledge engineer and knowledge editor. The knowledge engineer is responsible for structuring and developing the architecture of the knowledge base whilst the knowledge editor is responsible for adding and editing knowledge within the knowledge base. SemanticGuide can be deployed via a call centre version or a mobile version which is utilised by service technicians working underground in form of a PDA. SemanticGuide is also connected to various data sources such as oracle database for the knowledge base designer and Internet for web accessibility.
SemanticGuide is composed of three core features. These are the knowledge base (rules declaration), the knowledge model which consists of an ontology for knowledge representation and OntoBroker which acts as the semantic middleware inference engine.

\subsection{Adopted Knowledge Management Approach}

In order to develop the semantic application, it was crucial to adopt a knowledge management approach. Firstly, the identification of concepts types/hierarchy with key individuals at the sponsoring company was established. This was achieved through group discussions and the outcome was the selection of the appropriate case study that will be utilised to demonstrate the semantic application. The bicycle domain was chosen due to the fact that a bicycle possess over 80 mechanical parts which at anytime could become faulty and in need of resolution diagnosis. The idea is that if the semantic application could successful model a bicycle domain, it could then be useful for a more complex domain such as an aircraft.

The next stage was to acquire the experts' knowledge on bicycle maintenance. The experts' knowledge was in form of a repair manual for bicycles. This was developed by a group of experts within the domain. The advantage of utilising this manual as the basis of the application is that the knowledge represented within it has already been validated. This helped in ensuring that the semantic model contains validated knowledge.

Furthermore, UML use case diagrams and ontological knowledge representation techniques were used to model various aspects of the domain before the prototype development as explained later during this paper.

\subsubsection{Define Knowledge Area}

Defining a knowledge area is the responsibility of the knowledge manager. A knowledge area consists of concept product and problem. A concept bicycle was defined. The concept hierarchy was also defined seeing as different types of bicycles exist (road, mountain, BMX, etc). Furthermore, problems such as noise, chain failure, flat tyre, defective wheel, headset failure and pedal mounting failure can affect these bicycles. The use of taxonomy which is also considered as a semantic knowledge representation technique was used to establish the hierarchy of the created concepts. It was noticed that one of main advantages of SemanticGuide is that it can depict a systemic overview of the created concepts. However, it fails to illustrate a visual representation of the ontological model (relations between concepts). Furthermore, the semantic network itself mainly exists between the concepts. It does not exist between the rule definitions.

The definition of generic and specific knowledge areas suggests that problems can be resolved at lower levels of abstraction. Furthermore, attaching documents to symptoms of knowledge area enhances problem resolution and the definition of synonyms makes it easier for users to get along within the knowledge base. For example, noise was also defined as sound. Also, one could document experts' details (name, phone number, etc) in each knowledge area. This is effective if one is dealing with multiple experts and if the knowledge base being modelled is extremely complex. 

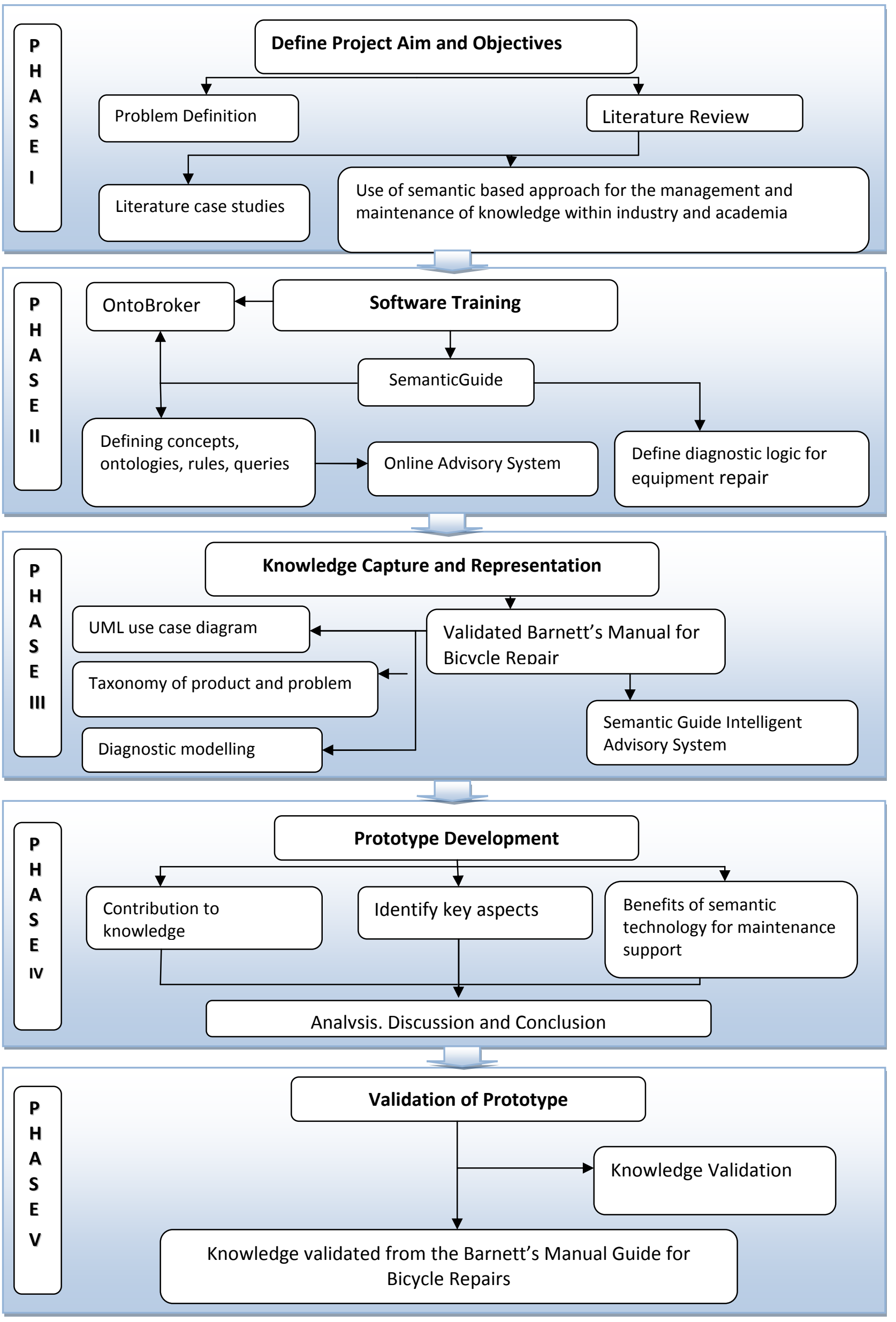

Figure 1 - Research Programme Methodology 


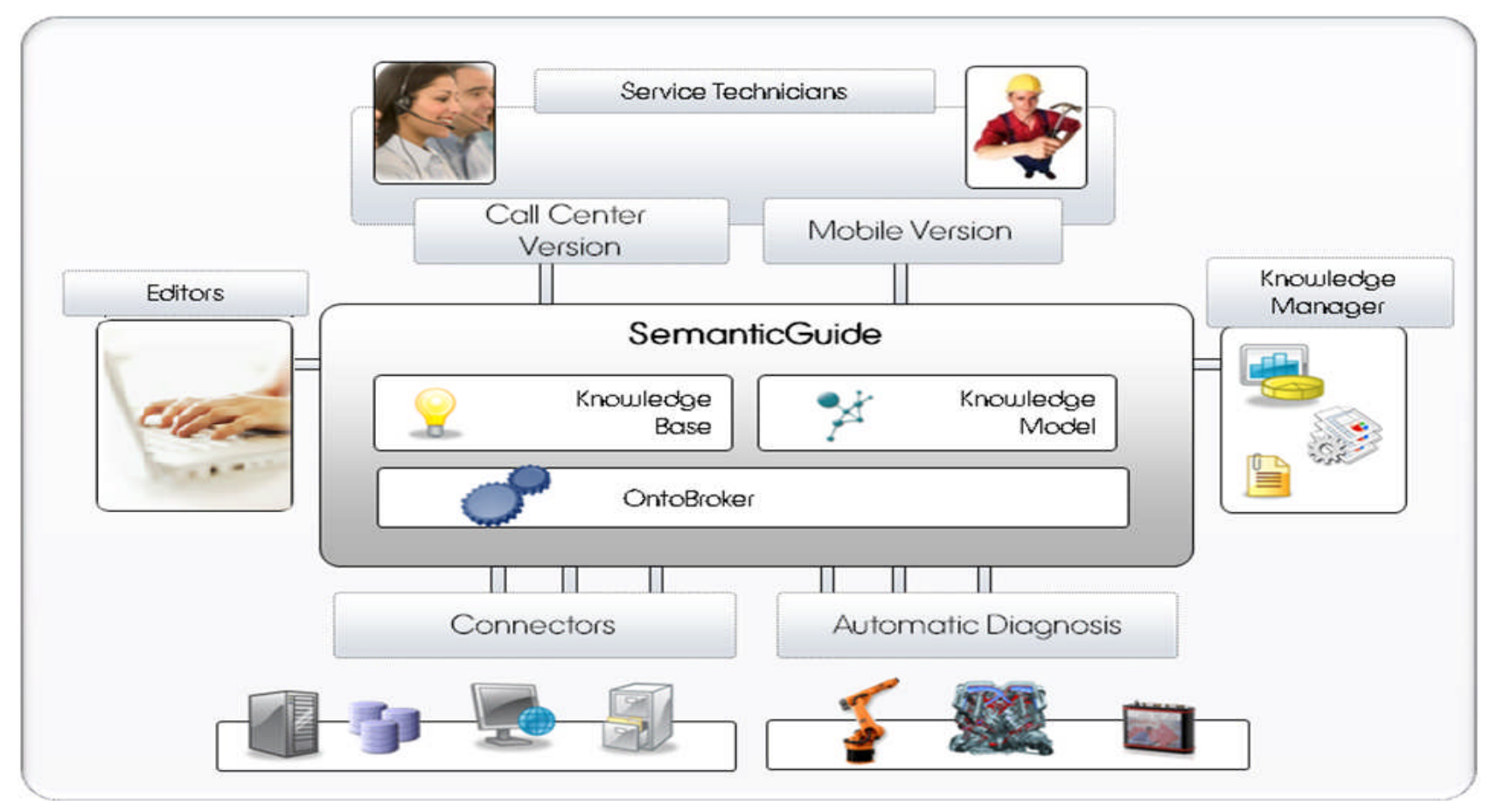

Figure 2 - SemanticGuide Architecture

\subsubsection{Add Symptoms and Solutions}

Once the knowledge area has been defined, the next step is to add symptoms and solutions for the defined knowledge area. A symptom is a characteristic or indication of a problem. A symptom has solutions which consist of causes and actions. It is essential to note that the solutions are recorded in order of frequency. This means the most common solution for a symptom occurrence within a knowledge area is recorded first within the knowledge base. The status quo of the knowledge base states that 40 symptoms have been included for the bicycle knowledge areas. An example of a resolution for a knowledge area is illustrated in Figure 3. The reusability of symptoms and solutions is one of the core advantages of SemanticGuide. The use of a global solution and question pool allows for re-usability of diagnosis questions and answers which saves time and effort. Furthermore, it was identified that the global pool of knowledge can be defined first instead of concepts creation. This functionality reinforces user-friendliness.

\subsubsection{Graphical Diagnosis Modelling}

The graphical diagnosis editor allows for the graphical representation of the diagnostic process modelling which adds clarity to an explanation and makes functionality editing easier. The GDE allows for the declaration of If/else rule statements which represents the diagnostic logic for each symptom within a knowledge area. In this process, it was useful to construct a small answering outline that suggests how the answering options are selected to guide the user up to the problem solution. There is also reusability of the stored questions within the knowledge base. Furthermore, every recorded solution within the knowledge base is saved in a global solution pool; therefore, it is possible to record solutions and add them to symptoms later. The modelling approach has proven that Semantic technology allows for declarative rules which are rules that can adapt to change of data rather than the traditional procedural rules. This means that rules can be defined without data availability and once the data is available, the rule automatically fires which is not the case for traditional rules declaration.

\subsubsection{Knowledge Base Export}

Once the knowledge base has been developed and the diagnostic process has been modelled, the next step is then to release the knowledge base for SemanticGuide.

A simple 3 step process is used to deploy the knowledge base designer and release the problem resolution functionality to the rest of the world. The first step is to create the update files, followed by creating the new knowledge base and lastly copying the knowledge base into the update folder. Furthermore, the knowledge base export process suggests that SemanticGuide can be deployed gradually which means it can mature with the platform. The knowledge base export process also automatically creates and sorts ontologies within the knowledge base for SemanticGuide to utilise

\subsubsection{SemanticGuide Online Advisory System}

Finally, once the knowledge base has been exported for SemanticGuide, the user can now interact and access the diagnosis process developed within the knowledge base. The modelled knowledge base is embedded within a user friendly interface offered by SemanticGuide. The diagnostic process logic guides the user to arrive at an appropriate solution and the user can select solution failure/success rates which act as input to the BIRT (business intelligence and reporting tools) analysis. This helps businesses identify which areas need focused improvement in order to maximise business performance. 


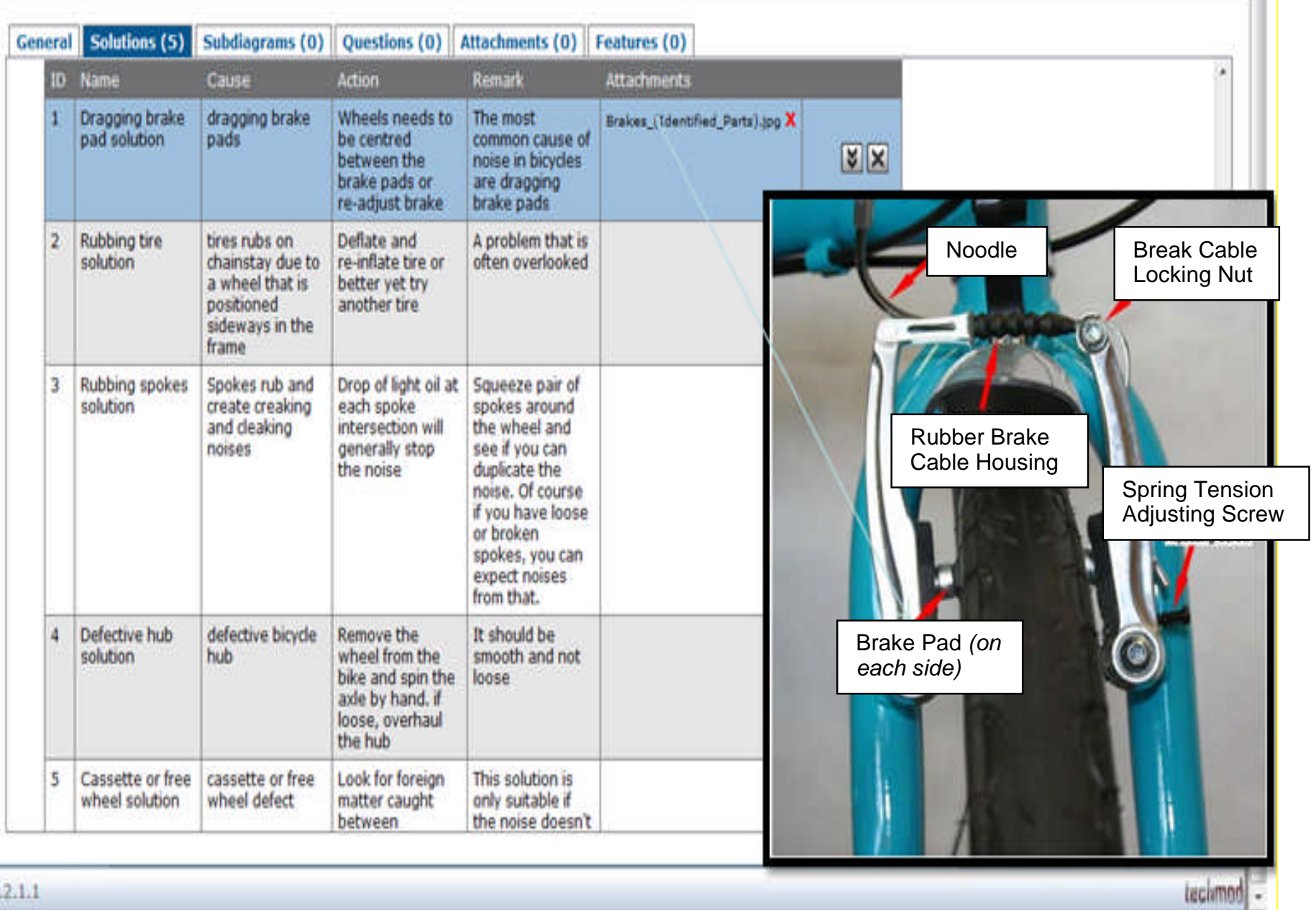

Figure 3 - Resolutions for knowledge area Bicycle/Noise

\section{VALIDATION}

During the development of the semantic based prototype, the validation of results and knowledge were also incorporated. One of the key strengths about the adopted methodology is that an expert was present during the development of the semantic based prototype. This helped in correcting and editing errors within SemanticGuide and ensured the verification and validation of knowledge within SemanticGuide for the bicycle maintenance support.

Furthermore, a standard bicycle manual repair document was used to model the problem domain. This explicit documented knowledge aided in validating the prototype. One can assume that the standards that created the model have already verified and validated the knowledge included within the standard. The bicycle manual was developed by a team of bicycle experts which reinforces the validation of this particular semantic based prototype.

This research project relied heavily on the 'Barnett's' manual repair for bicycle maintenance and an expert. For this purpose, it is imperative to identify the difficulty of transforming this knowledge source into a semantic based platform. This is due to the complexity of capturing and understanding various knowledge forms.

Although, the bicycle manual was explicitly documented, it was challenging to acquire the knowledge needed to develop the diagnostic logic for each knowledge area. It was vital for the researcher to adopt a qualitative approach in observing and understanding the know-how of the expert's knowledge which enabled the accurate capture and implementation of the semantic based prototype for product maintenance support.

\section{CONCLUSIONS}

The development of a semantic based prototype to enhance the management and maintenance of knowledge has been demonstrated. There is a need to efficiently and effectively manage knowledge resources through the use of semantics and ontologies. In order to successfully construct this practical case study, the UML use case diagram was used to illustrate how the system (actors) interacts with the external environment. This helped in identifying the roles, functionalities and boundaries within SemanticGuide. Furthermore, the use of the taxonomy knowledge representation technique established the generalisation/specialisation hierarchical construction within SemanticGuide. As stated earlier, one of the main disadvantages of the application is that it fails to depict the ontological representation of the knowledge base and simply tabulates the concepts hierarchy within the domain. Furthermore, the semantic relations are only defined between the developed concepts. The semantic 
application fails to define the ontological relations between the rule definitions. However, one of the main benefits of using this semantic technology is that it offers reusability and maintainability. The re-usability of concepts and rules are an imperative factor in use of semantic technology. This has been identified within SemanticGuide when adding symptoms and solutions to the knowledge base and during the modelling of the graphical rules.

The developed semantic based prototypes also proved that semantic technology is dynamic in nature. It adapts and changes over time. The functionalities within SemanticGuide can be deployed gradually which means that it can mature with the platform. This is achieved through the knowledge base export functionality as explained earlier.

Semantic technology also allows for declarative rules which are rules that can adapt to change of data rather than the traditional procedural rules as explained in the graphical diagnosis rule modelling section.

Furthermore, it was noticed through critical analysis that the use of semantic application is very similar to that of object oriented programming. The main three of OOP are inheritance, encapsulation and polymorphism. Semantics has the capability to portray these three key features. However, semantic technology is different because of the use of synonyms which allows one or more words to be interchanged within a context. This gives semantic technology an advantage over the OOP paradigm. An example of this was illustrated earlier when noise was also defined as sound within a knowledge area of SemanticGuide.

Finally, this research project has quantified the use and benefits of semantic technology to manage knowledge. This technology is highly scalable and can be used to model a more complex domain such as maintenance of an aircraft. Moreover, such a complex domain will contain thousands of declarative rules. However, Semantic technology has proven it can adapt to such complexity and can be applied to any industrial field due to its dynamic, adaptive and evolving nature.

\section{ACKNOWLEDGMENTS}

The authors would like to thank TechModal Ltd for sponsoring this project. They would like also to express their gratitude to all the persons who contributed directly or indirectly to the success of the project.

\section{REFERENCES}

[1] Grover, V., \& Davenport, T. H. (2001). General Perspective on Knowledge Management: Fostering a Research Agenda. Journal of Management Information Systems , 5-21.

[2] NHS Evidence, (2002)"KM Technology, IT and knowledge management" [Online] Available at: http://www.library.nhs.uk/KnowledgeManagemen $\mathrm{t} /[$ Accessed 21st May 2009]

[3] Milton, N. (2007). Knowledge Acquisition in Practice: A Step-by-Step Guide. London: Springer.

[4] O'Hara, K., Shadbolt, N., (2001) Managing Knowledge Capture: Economic, Technological and Methodological Considerations. Technical Report, Dept of Electronics \& Computer Science, University of Southampton.

[5] Darlington, K., (2000), "The essence of expert systems", essence of computing, Southbank University, Edinburgh: Pearson Education
Limited

[6] Ulises, C., Ignasi, R., Campos, M., Rollón, C. (2003), "current reasoning engine practice and integration strategies", RIMSAT, University of Girona: 2003

[7] Kingston, J., (2004), "Conducting feasibility study for knowledge-based systems", Knowledge-based Systems, Vol. 17 No 2-4, pp. 157-64.

[8] Metaxiotis, K., (2004), "RECOT: an expert system for the reduction of environmental cost in the textile industry", Information Management \& Computer Security, Vol. 12 No. 3, pp. 2182731.

[9] Horn, W., Popow, C., Miksch, S., Seyfang, A., (2002), "Benefits of a knowledge-based system for parenteral nutrition support: a report after 5 years of routine daily use", Proceedings of 15th European Conference on Artificial Intelligence (ECAI 2002), IOS Press, Amsterdam, pp. 613-7

[10] Reimer, U., Margelisch, A. and Staudt, M., (2000), "EULE: a knowledge-based system to support business processes", Knowledge-based Systems, Vol. 13 No. 5, pp. 261-9.

[11] Nedovic, L., Devedzic, V., (2003), "DEVEX: an expert system for currency exchange advising", International Journal of Knowledge-Based Intelligent Engineering Systems, Vol. 7 No. 1, pp. 38-45.

[12] Barski, C., (2003), "How to tell stuff to a computer", The Enigmatic art of knowledge representation [Online], Available at: www.lisperati.com/tellstuff/index.html [Accessed: 25/06/09]

[13] Uschold, M., (2002), "A semantic continuum on the semantic web", The Knowledge Engineering Review (2002), 17:1:87-91Cambridge University Press

[14] Horrocks, I., (2008), "Semantic Web", HumanComputer Interaction Series, chapter 19, pages 315-330, 1st edition, Springer: London, September 2008.

[15] Davies, J., Fensel, D., Harmelen, F.V., (2003) "Towards the Semantic Web: Ontology-driven Knowledge Management", John Wiley \& Sons, Inc., New York, NY, 2003

[16] NMK, (2009), Industry Mixed on Development of Semantic Web [Online] Available at:http://www.nmk.co.uk/article/2009/4/6/industry -mixed-on-development-of-semantic-web [Accessed 25th June 2009]

[17] Agorastos, T., Koutkias, V., Falelakis, M., (2009), "Semantic Integration of Cervical Cancer Data Repositories to Facilitate Multicenter Association Studies": The ASSIST Approach, Cancer Informatics 2009: 831 - 44

[18] AXONomics, (2008), "Financial industry use of semantic technology", Available at: http://www.secondintegral.com/axonomics/?p=4 8 [Accessed: 01/06/2008]

[19] Yam, R. C. M., Tse, P.W., Li, L., and Tu, P., (2001), "Intelligent Predictive Decision Support System for Condition-Based Maintenance", the International Journal of Advanced Manufacturing 
Technology, vol. 17, no 5, February, pp. 383391.

[20] Fuchs, F., Henrici, S., Pirker, M., Berger, M., Langer, G., Seitz, C., (2006), "Towards Semantics-based Monitoring of Large-Scale Industrial Systems", International Conference on Intelligent Agents, Web Technologies and Internet Commerce, November, pp 261-261

[21] TechModal., (2009)., About Us., Available at:http://www.techmodal.com/webpages/about.ht $\mathrm{ml}$ [Accessed: 10/January/2010]

[22] Ontoprise., (2009)., SemanticGuide: Intelligent Advisory System to Support Complex Consuilting Proceses. [Online] Available at: http://www.ontoprise.de/en/home/products/sema nticguide/ [Accessed: 12/January/2010] 